\title{
A LIFTING THEOREM FOR THE TIME REGULARITY OF SOLUTIONS TO ABSTRACT EQUATIONS WITH UNBOUNDED OPERATORS AND APPLICATIONS TO HYPERBOLIC EQUATIONS
}

\author{
I. LASIECKA AND R. TRIGGIANI
}

(Communicated by Walter Littman)

ABSTRACT. We consider the solution operator

$$
(L u)(t)=A \int_{0}^{t} G(t-\tau) A^{-1} B u(\tau) d \tau
$$

corresponding to the abstract equation $\dot{x}=A x+B u$ on a reflexive Banach space $X$, where the linear operator $A: X \supset \mathscr{D}(A) \rightarrow X$ is the infinitesimal generator of a (strongly continuous) group $G(t)$ of bounded operators on $X$, and $B: U \supset \mathscr{D}(B) \rightarrow X$ is a generally unbounded linear operator with $A^{-1} B \in \mathscr{L}(U, X), U$ being another reflexive Banach space (without loss of generality we take $A$ to be boundedly invertible). Let $0<T<\infty$ be given. We prove the following theorem: if $L$ is continuous $L^{p}(0, T ; U) \rightarrow L^{p}(0, T ; X)$, $1<p<\infty$, then in fact $L$ : continuous $L^{p}(0, T ; U) \rightarrow C([0, T] ; X)$, a lifting regularity theorem in the time variable. Moreover, we show by a parabolic example with nonhomogeneous term in the Dirichlet boundary conditions that the theorem fails to be true, if $G(t)$ is merely a s.c. semigroup even if holomorphic. Applications of the theorem include mixed hyperbolic problems, including second order scalar hyperbolic equations defined on an open bounded domain $\Omega \subset R^{n}, \partial \Omega=\Gamma$, with nonhomogeneous term of class $L^{2}\left(0, T ; L^{2}(\Gamma)\right)$ acting in the Dirichlet or in the Neumann boundary conditions. In the former case, the theorem recovers the authors' original procedure which yielded optimal regularity results for this dynamics [L-T.2]; in the latter, the theorem improves upon results of Lions-Magenes [L-M.1, vol. II]. Extension to $T=\infty$ is also studied.

1. Introduction and main result. Let $\Omega \subset R^{n}, n \geq 1$, be an open bounded domain with sufficiently smooth boundary $\Gamma$. Consider the following hyperbolic mixed problem described by a second order hyperbolic scalar equation in the unknown $y(t, x), x \in \Omega$, with nonhomogeneous term $u(t, \sigma), \sigma \in \Gamma$, in the Dirichlet boundary conditions and, say, zero initial conditions

$$
\begin{array}{ll}
y_{t t}=\mathscr{Q}(x, \partial) y & \text { in } Q_{T} \equiv(0, T] \times \Omega \\
y(0, x) \equiv y_{0}=y_{t}(0, x) \equiv y_{1}=0 & \text { in } \Omega ; \\
y(t, \sigma)=u(t, \sigma) \in L^{2}\left(0, T ; L^{2}(\Gamma)\right) \equiv L^{2}\left(\Sigma_{T}\right), & \Sigma_{T} \equiv(0, T] \times \Gamma
\end{array}
$$

Here, $-\mathscr{Q}(x, \partial)$ is an arbitrary second order, uniformly elliptic operator (in $\bar{\Omega}$ ) with say, symmetric principal part, whose coefficients may depend on the space

Received by the editors June 22, 1987.

1980 Mathematics Subject Classification (1985 Revision). Primary 47A50, 35L20.

Research partially supported by the National Science Foundation under Grant NSF-DMS 8301668. 
variable $x$, but not on the time variable $t$. Canonically, we have $\mathscr{Q}(x, \partial)=\Delta$, the $n$-dimensional Laplacian in $x$. The authors' original proof [L-T.1, L-T.2] of the following regularity result

$$
\mathscr{L}_{T}: u \rightarrow\left[y, y_{t}\right]: \text { continuous } L^{2}\left(\Sigma_{T}\right) \rightarrow C\left([0, T] ; L^{2}(\Omega) \times H^{-1}(\Omega)\right)
$$

for any $0<T<\infty$-which improved by approximately " $\frac{1}{2}$ " in Sobolev space order the space regularity of previously known results [L-M.1, I, II]-was carried out through the following two step-procedure.

(1) First it was shown that [L-T.1],

$$
\mathscr{L}_{T} \text { : continuous } L^{2}\left(\Sigma_{T}\right) \rightarrow L^{2}\left(0, T ; L^{2}(\Omega) \times H^{-1}(\Omega)\right) .
$$

This result was obtained in the general case by using pseudo-differential operator techniques (as well as Fourier analysis techniques on the direct eigenfunction expansions for the solution, in the canonical case of the Laplacian $\Delta$ on special geometries where $\Omega$ is either a parallelepiped or else a sphere).

(2) Subsequently, the regularity result in (1.2) was 'lifted' up in time to the regularity result in (1.1), [L-T.2], by making explicit use of (1.2). This step was carried out by purely functional analytic or operator theoretic techniques, as it relied on an abstract operator model for the second order mixed problem in $y$, first introduced in [T.1], further pursued in [L-T.1], and extensively used since by the authors and by others in boundary control problems for hyperbolic equations, see [L-T.3] and references therein. This model is based on cosine-sine operator theory on $L^{2}(\Omega)$ [and $H^{-1}(\Omega)$ ] (see (1.22) below) and hence admits a semigroup (group) version on $L^{2}(\Omega) \times H^{-1}(\Omega)$, noted explicitly, say in [L-T.3, DaP-L-T.1] etc. and also below in Example 1. A crucial ingredient in Step 2 is the following equivalence of 'interior' and 'trace' regularity, which once translated from the "operator framework" to the "p.d.e. framework" for the hyperbolic mixed problem (1.0) reads as follows:

$$
\mathscr{L}_{T}: \text { continuous } L^{2}\left(\Sigma_{T}\right) \rightarrow L^{2}\left(0, T ; L^{2}(\Omega) \times H^{-1}(\Omega)\right)
$$

if and only if

$$
\left\{\Phi_{0}, \Phi_{1}\right\} \rightarrow \frac{\partial \Phi}{\partial \nu_{Q^{*}}}: \text { continuous } H_{0}^{1}(\Omega) \times L^{2}(\Omega) \rightarrow L^{2}\left(\Sigma_{T}\right)
$$

where $\partial / \partial \nu_{Q^{*}}$ is the conormal derivative with respect to the adjoint problem described by $\mathscr{Q}^{*}\left[\right.$ hence $\partial / \partial \nu_{Q^{*}}=\partial / \partial \nu$ when $\mathscr{Q}=\Delta$ ]. Here $\Phi$ solves the corresponding homogeneous problem

$$
\begin{aligned}
& \Phi_{t t}=\mathscr{Q}^{*} \Phi \text { in } Q_{T} ; \\
& \Phi(0, x)=\Phi_{0} \in H_{0}^{1}(\Omega), \quad \Phi_{t}(0, x)=\Phi_{1} \in L^{2}(\Omega) ; \\
& \Phi \equiv 0 \text { on } \Sigma_{T} .
\end{aligned}
$$

The operator formulation of (1.3) (see (1.23) below, Example 1) then allowed for an operator argument in [L-T.2] to improve the regularity of $\mathscr{L}_{T}$ from (1.2) to (1.1).

At the time of writing [L-T.2] it was recognized that (i) the operator approach given there, although tailored and specialized to the cosine-sine operator model for second order hyperbolic equations of interest there, works equally well in full generality in the context of dynamics that are described by an arbitrary s.c. (strongly continuous) group of operators in an arbitrary reflexive Banach space; (ii) moreover, the improvement in the time regularity that one obtains goes in general from 
$L^{p}(0, T)$ to $C[0, T], 1<p<\infty$, and not merely for the case $p=2$ as used (and needed) in [L-T.2]. The validity of such a more general and abstract result has been used freely by the authors in correspondence, private talks ${ }^{1}$ or talks at conferences, etc. but never put in print. As it stands, [L-T.2] apparently may give the impression that the question of the generality of the "lifting" argument from $L^{p}(0, T)$ to $C[0, T]$ (while preserving the space regularity) is called for further investigation, beyond the specific case of [L-T.2]; see [W.1].

Accordingly, in this note we wish to formalize our original argument in [L-T.2] in an abstract general result.

Let $X$ be a reflexive Banach space, let $A: X \supset \mathscr{D}(A) \rightarrow X$ be a linear (closed, densely defined) operator, assumed to be the generator of a strongly continuous (s.c.) group $G(t)$ of operators on $X$. Let $U$ be another reflexive Banach space and $B$ a (generally unbounded) linear operator $U: \supset \mathscr{D}(B) \rightarrow X$ such that $A^{-1} B \in$ $\mathscr{L}(U, X)$, the Banach space of all bounded linear operators from $U$ to $X$ (without loss of generality for the problem here considered, we can assume $0 \in \rho(A)$, the resolvent set of $A$, so that $A^{-1} \in \mathscr{L}(X)$ ). Consider the operator $L_{T}$ defined by

$$
\left(L_{T} u\right)(t)=A \int_{0}^{t} G(t-\tau) A^{-1} B u(\tau) d \tau
$$

(conventionally written as $\int_{0}^{t} G(t-\tau) B u(\tau) d \tau$ ) formally associated to the abstract equation

$$
\dot{x}=A x+B u, \quad x(0)=x_{0}=0 .
$$

Our main result is

THEOREM. Assume that

$$
L_{T}: \text { continuous } L^{p}(0, T ; U) \rightarrow L^{p}(0, T ; X), \quad 1<p<\infty,
$$

for some $0<T<\infty$, and hence all $0<T<\infty$ (by a change of variable and semigroup property). Then, in fact

$$
L_{T}: \text { continuous } L^{p}(0, T ; U) \rightarrow C([0, T] ; X) .
$$

PROOF (AFTER [L-T.2]).

Step 1. The adjoint $L_{T}^{*}$ of $L_{T}$ in the sense that with $1 / p+1 / q=1$ [H.1, p. 89],

$$
\left(L_{T} u, f\right)_{p, X ; q, X^{*}}=\left(u, L_{T}^{*} f\right)_{p, U ; q ; U^{*}}
$$

is given by

$$
\left(L_{T}^{*} f\right)(t)=\int_{t}^{T} B^{*} G^{*}(\tau-t) f(\tau) d \tau
$$

and satisfies

$$
L_{T}^{*}: \text { continuous } L^{q}\left(0, T ; X^{*}\right) \rightarrow L^{q}\left(0, T ; U^{*}\right),
$$

\footnotetext{
${ }^{1}$ During the workshop on "Control of systems governed by partial differential equations" held in Val David, Quebec, Canada, October 5-9, 1986, C. Dafermos (Brown University) has informed one of the authors (R.T.) that he also has a proof (unpublished) of the lifting theorem presented here, which we understand to be a 'direct' proof, rather than by duality as the one given here.
} 
where the notation on the left and on the right of $(1.8 \mathrm{a})$ denotes, respectively, the duality pairing between $L^{p}(0, T ; X)$ and $L^{q}\left(0, T ; X^{*}\right)$, and between $L^{p}(0, T ; U)$ and $L^{q}\left(0, T ; U^{*}\right)$.

Step 2. Apply $L_{T}^{*}$ to a special $f$, given by the dual free $(B \equiv 0)$ dynamics of (1.5) backward in time ${ }^{2}$; i.e. to $\hat{f}()$ given by

$$
\hat{f}(\tau) \equiv G^{*}(-\tau) x^{*} \in L^{q}\left(0, T ; X^{*}\right), \quad x^{*} \in X^{*} .
$$

Then

$$
\left(L_{T}^{*} \hat{f}\right)(t)=B^{*} G^{*}(-t) x^{*}(T-t) \in L^{q}\left(0, T ; U^{*}\right)
$$

for any finite $T$, and hence

$$
\left(L_{T}^{*} \hat{f}\right)(t)=B^{*} G^{*}(-t) x^{*} \in L^{q}\left(0, T ; U^{*}\right), \quad x^{*} \in X^{*},
$$

continuously; i.e. writing $x^{*}=G^{*}(T) y^{*}, y^{*}=G^{*}(-T) x^{*} \in X^{*}$ we have that

$$
J \equiv B^{*} G^{*}(T-t): \text { continuous } X^{*} \rightarrow L^{q}\left(0, T ; U^{*}\right) .
$$

[Note that at this stage, for $u \in L^{p}(0, T ; U)$ we have

$$
\begin{aligned}
\left(u, J x^{*}\right)_{p, U ; q, U^{*}} & =\int_{0}^{T}\left(u(t),\left(J x^{*}\right)(t)\right)_{U ; U^{*}} d t \\
& =\int_{0}^{T}\left(u(t), B^{*} G^{*}(T-t) x^{*}\right)_{U ; U^{*}} d t=\left(z_{T, u}, x^{*}\right)_{X ; X^{*}}
\end{aligned}
$$

for a unique element $z_{T, u} \in X$, since the well-defined expression on the left of (1.13) defines a continuous linear functional on $X^{*}$; the new notation in (1.13) denotes the duality pairing between $U$ and $U^{*}$, and between $X$ and $X^{*}$. Then one computes

$$
\left(z_{T, u}, x^{*}\right)_{X ; X^{*}}=\left(\int_{0}^{T} G(T-t) B u(t) d t, x^{*}\right)_{X ; X^{*}}=\left(J^{*} u, x^{*}\right)_{X ; X^{*}}
$$

first for $x^{*} \in \mathscr{D}\left(A^{*}\right)$ and then for all $x^{*} \in X^{*}$ by continuous extension. Thus, the adjoint $J^{*}$ of $J$ in (1.12) is

$$
\left.J^{*} u=\int_{0}^{T} G(T-t) B u(t) d t: \text { continuous } L^{p}(0, T ; U) \rightarrow X\right] .
$$

Step 3. With $v \in L^{1}\left(0, T ; X^{*}\right)$ and $u \in L^{p}(0, T ; U)$ we compute from (1.4)

$$
\begin{gathered}
\int_{0}^{T}\left(L_{T} u(t), v(t)\right) d t=\int_{0}^{T} \int_{0}^{t}\left(u(\tau), B^{*} G^{*}(t-\tau) v(t)\right)_{p, U ; q, U^{*}} d \tau d t \\
\quad \leq \int_{0}^{T}\left\{\int_{0}^{t}|u(\tau)|_{U}^{p} d \tau\right\}^{1 / p}\left\{\int_{0}^{t}\left|B^{*} G^{*}(t-\tau) v(t)\right|_{U^{*}}^{q} d \tau\right\}^{1 / q} d t
\end{gathered}
$$

(replacing $t$ with $T$ in both integral signs and using (1.12))

$$
\leq \int_{0}^{T} C_{T}|v(t)|_{X^{*}} d t\|u\|_{L^{p}(0, T ; U)}=C_{T}|v|_{L^{1}\left(0, T^{*} X^{*}\right)}|u|_{L^{p}(0, T ; U)} .
$$

\footnotetext{
${ }^{2}$ This step corresponds to $L^{*} C^{*}(\cdot) x$ and $L^{*} Q^{* 1 / 2} S^{*}(\cdot) x$ in [L-T.2] in the notation of [L-T.2], for the mixed problem (1.0).
} 
Thus by the closed graph theorem,

$$
L_{T} \text { : continuous } L^{p}(0, T ; U) \rightarrow L^{\infty}(0, T ; X) .
$$

Step 4. By taking now $u_{n}$ smooth, say $u_{n} \in C^{1}([0, T] ; U)$ with $u_{n} \rightarrow u$ in $L^{p}(0, T ; U)$, and integrating $\left(L_{T} u_{n}\right)(t)$ by parts, we then improve the continuity in (1.17) to the continuity in (1.7).

REMARK 1. The assumption that $G(t)$ be a s.c. group is crucial in the above theorem, in the sense that if $G(t)$ is only a s.c. semigroup even if holomorphic, the conclusion of the theorem is false. As an example illustrating this, let $z(t, x)$ be the solution of a corresponding parabolic equation with, say zero initial condition and with forcing term $u$ in the Dirichlet boundary conditions; say $z_{t t}=\Delta z$ in $Q_{T} ; z(0, x) \equiv 0 ; z(t, \sigma)=u(t, \sigma)$ on $\Sigma_{T}$. Then the corresponding free solution $(u \equiv 0)$ is described by a s.c., holomorphic semigroup on $L^{2}(\Omega)$ (which therefore is not a group). We have that the map $u \rightarrow y$ is continuous from $L^{2}\left(\Sigma_{T}\right) \rightarrow L^{2}\left(Q_{T}\right)$ (even $\rightarrow L^{2}\left(0, T ; H^{1 / 2}(\Omega)\right.$ ), yet the map $u \rightarrow y(T)$ from $L^{2}\left(\Sigma_{T}\right)$ to $L^{2}(\Omega)$ is not continuous. In fact, for a preassigned $0<T<\infty$, one may construct $u \in L^{2}\left(\Sigma_{T}\right)$ whose corresponding solution $y$ satisfies $y(T) \notin L^{2}(\Omega)[\mathbf{L - 3}$, p. 202], even in the one-dimensional case.

\section{Applications to hyperbolic equations.}

EXAMPLE 1. For the second order mixed problem (1.0) in $y$ we have the following specialization of the situation described by the theorem. We let $X=L^{2}(\Omega) \times$ $H^{-1}(\Omega), x=\left[y, y_{t}\right] ; U=L^{2}(\Gamma)$. Moreover, with $\mathscr{Q}$ denoting the operator that realizes $\mathscr{Q}(x, \partial)$ with homogeneous Dirichlet boundary conditions on $L^{2}(\Omega)$, we have

$$
A=\left|\begin{array}{cc}
0 & I \\
\mathscr{Q} & 0
\end{array}\right| ; \quad B u=\left|\begin{array}{c}
0 \\
-\mathscr{Q} D u
\end{array}\right| \text { (formally); } \quad A^{-1} B=\left|\begin{array}{c}
-D u \\
0
\end{array}\right| .
$$

$D$ (Dirichlet map) defined by $D h=v$ meaning: $\mathscr{Q}(x, \partial) v=0$ in $\Omega, v=h$ on $\Gamma$; also

$$
G(t)=\left|\begin{array}{cc}
C(t) & S(t) \\
-\mathscr{Q} C(t) & C(t)
\end{array}\right|
$$

where $C(t)$ is the s.c. cosine operator generated by $-\mathscr{Q}$ and $S(t)=\int_{0}^{t} C(\tau) d \tau$; moreover with $x=\left[x_{1}, x_{2}\right]$ we have

$$
\begin{gathered}
B^{*}\left|\begin{array}{l}
x_{1} \\
x_{2}
\end{array}\right|=D^{*} \mathscr{Q}^{* 1 / 2} \mathscr{Q}^{-1 / 2} x_{2}, \quad \text { with dense domain in } X, \\
B^{*} G^{*}(T-t)\left|\begin{array}{l}
x_{1} \\
x_{2}
\end{array}\right|=D^{*} \mathscr{Q}^{*} S^{*}(t) x_{1}+D^{*} C^{*}(t) x_{2} .
\end{gathered}
$$

Finally

$$
\left(L_{T} u\right)(t)=\left|\begin{array}{ll}
\mathscr{Q} & \int_{0}^{t} S(t-\tau) D u(\tau) d \tau \\
\mathscr{Q} & \int_{0}^{t} C(t-\tau) D u(\tau) d \tau
\end{array}\right|
$$

and we have recovered the model of $[\mathbf{T . 1}, \mathbf{L}-\mathbf{T} .1]$ for the second order hyperbolic mixed problem. Since $D^{*} \mathscr{Q}^{*}=\partial / \partial \nu_{Q^{*}}$ (see [L-T.1]), then, by $(1.21)$, statement (1.12) rewritten as

$$
\frac{\partial \Phi}{\partial \nu_{\mathbb{Q}^{*}}}=D^{*} A^{*}\left[S^{*}(t) x_{1}+C^{*}(t) \mathscr{Q}^{*-1} x_{2}\right]
$$

corresponds to statement (1.3). 
REMARK 1.2. The subsequent treatment [L.1, L-L-T.1], of the hyperbolic mixed problem (1.0) markedly simplified the original proof of (1.1) outlined in Steps (1) and (2) above. In this new approach, one first proves directly the trace theory result (1.3) by means of a p.d.e. multiplier technique (using the multiplier $h \cdot \nabla \Phi$ on the $\Phi$-hyperbolic problem below (1.3) with $h(x)$ a $C^{1}(\bar{\Omega})$-vector field with $h=\nu$ on $\Gamma$ ). Since (1.3) corresponds abstractly to statement (1.12) (see (1.23) above, Example 1), the ad hoc version (using cosine-sine operator theory) of Step 3 of the proof above permits one to obtain the desired result (1.1).

This same multiplier technique has allowed to show that, in fact, for all $T>$ some explicitly computed $T_{0}>0$, with $T_{0}$ depending on the geometry of $\Omega$, one also has the converse of (1.3); i.e.:

$$
\int_{\Sigma}\left(\frac{\partial \Phi}{\partial \nu_{\mathscr{Q} *}}\right)^{2} d \Sigma \geq C\left(T-T_{0}\right)\left\|\left\{\Phi_{0}, \Phi_{1}\right\}\right\|_{H_{0}^{1}(\Omega) \times L^{2}(\Omega)}^{2}
$$

(see [H.1, L-T.4]), a crucial result for showing exact controllability of the hyperbolic mixed problem $(1.0)$ in $\left[y, y_{t}\right]$ on the space $L^{2}(\Omega) \times H^{-1}(\Omega)$ [L.2, T.2].

EXAMPLE 2. The Neumann problem: $y_{t t}=\mathscr{Q}(x, \partial) y$ in $Q_{T} ; y(0, x)=y_{t}(0, x)=$ 0 in $\Omega ; \partial y / \partial \nu_{\mathscr{Q}}=u \in L^{2}\left(\Sigma_{T}\right)$ provides another example.

The choice of the space depends on the domain $\Omega$. For general domains $\Omega$, the classical result of [L-M.1, vol. II, p. 120],

$$
\left.u \rightarrow\left[y, y_{t}\right]: \text { continuous } L^{2}\left(\Sigma_{T}\right) \rightarrow L^{2}\left(0, T ; H^{1 / 2}(\Omega)\right) \times H^{-1 / 2}(\Omega)\right)
$$

can be improved using our preceding Theorem here to read

$$
u \rightarrow\left[y, y_{t}\right]: \text { continuous } L^{2}\left(\Sigma_{T}\right) \rightarrow C\left([0, T] ; H^{1 / 2}(\Omega) \times H^{-1 / 2}(\Omega)\right) .
$$

This was done using the ad hoc specialized version of the preceding Theorem (in the style of [L-T.2]) in (L-T.5]. Hence, in this case, $X=H^{1 / 2}(\Omega) \times H^{-1 / 2}(\Omega)$. Recent investigations [L-T.6] show that this result is not optimal in the space regularity, which in fact can be improved, for arbitrary (smooth) domains, to $X=$ $H^{3 / 5-\varepsilon}(\Omega) \times H^{-2 / 5-\varepsilon}(\Omega)$. The specialized versions

$$
\begin{aligned}
& u \rightarrow\left[y, y_{t}\right]: \text { continuous } L^{2}\left(\Sigma_{T}\right) \rightarrow L^{2}\left(0, T ; H^{\alpha}(\Omega) \times H^{\alpha-1}(\Omega)\right), \\
& \alpha=3 / 4-\varepsilon, \varepsilon>0 \text {, for } \Omega \text { a parallelepiped; } \\
& \alpha=2 / 3 \text { for } \Omega \text { a sphere, }
\end{aligned}
$$

which were shown in [L-T.1] were lifted later in [L-T.2], to read

$$
u \rightarrow\left[y, y_{t}\right]: \text { continuous } L^{2}\left(\Sigma_{T}\right) \rightarrow C\left([0, T] ; H^{\alpha}(\Omega) \times H^{\alpha-1}(\Omega)\right)
$$

whereby now $X=H^{\alpha}(\Omega) \times H^{\alpha-1}(\Omega)$. The abstract model for the Neumann case is similar to the one given in Example 1 in the Dirichlet case, except that the Dirichlet map $D$ is now replaced by the Neumann map $N=N_{\lambda_{0}}$ defined by $N h=v$ meaning: $\left[\mathscr{Q}(x, \partial)+\lambda_{0}\right] v=0$ in $\Omega ; \partial v / \partial \nu_{\mathscr{Q}}=h$ on $\Gamma$, where $\lambda_{0}$ (real) operates a suitable translation so that this elliptic problem has a unique (generalized) solution. For instance, if $\mathscr{Q}(x, \partial)=\Delta$, we may take say $\lambda_{0}=1$. We omit details and refer e.g. to $[\mathbf{L}-\mathbf{T . 1}, \mathbf{T . 3}]$.

As a final example one may consider first order hyperbolic systems where now the preceding Theorem with $X=L^{2}(\Omega)$ re-proves the improvement from $L^{2}\left(0, T ; L^{2}(\Omega)\right)$ 
of $[\mathbf{K . 1}]$ to $C\left([0, T] ; L^{2}(\Omega)\right)$ of $[\mathbf{R . 1}]$, at least in the case of the free dynamics being generated by a group (this depends on the boundary conditions, see [R.2]). Details of the abstract model are omitted here and refereed to in [C-1, C-L.1].

Finally, we remark that some Euler-Bernoulli mixed problems also satisfy the abstract 'trace theory' result (1.12), hence (1.7). with $p=2$ in fact, on appropriate function spaces. See [F-L-T.1, Appendix C, L-T.9].

3. Continuity of $L$ from $L^{p}(0, \infty ; U)$ to $L^{p}(0, \infty ; X)$. In this section we present another regularity property of the abstract equation (1.5), which extends the continuity of the map defined by (1.6) from the case where $T$ is finite to the case where $T=\infty$. More precisely, throughout this section the hypotheses are as follows.

$\left(\mathrm{H}_{1}\right)$ The spaces $U$ and $X$ are reflexive Banach spaces and $A$ is now merely the infinitesimal generator of a strongly continuous semigroup $e^{A t}$ on $X, t \geq 0$, (which may not be a group), which is moreover assumed to be uniformly stable; i.e. there are constants $M \geq 1$ and $\omega>0$ such that

$$
\left\|e^{A t}\right\|_{\mathscr{L}(X)} \leq M e^{-\omega t}, \quad t \geq 0 .
$$

$\left(\mathrm{H}_{2}\right)$ The operator $B$ satisfies $A^{-1} B \in \mathscr{L}(U, X)$ as in $\S 1$ and for $1<p<\infty$, the map

$$
\left\{\begin{array}{l}
u \rightarrow \int_{0}^{T} e^{A(T-t)} B u(t) d t \\
\text { is continuous } L^{p}(0, T ; U) \rightarrow X
\end{array}\right.
$$

for some $T<\infty$ (hence for $2 T, 3 T$, etc. by a change of variable); i.e. there is a constant $c_{T}>0$ such that

$$
\left\|\int_{0}^{T} e^{A(T-t)} B u(t) d t\right\|_{X} d t \leq c_{T}\left\{\int_{0}^{T}\|u(t)\|_{U}^{p} d t\right\}^{1 / p} .
$$

From now on we shall drop the subscripts $X, U$ on the norms as no confusion will arise.

REMARK 3.1. It follows by duality (as in (1.12)-(1.15) of $\S 1$ ) that assumption $\left(\mathrm{H}_{2}\right)$ is equivalent to the statement that the operator $B^{*} e^{A^{*} t}$ admits a continuous extension from $X^{*}$ to $L^{q}\left(0, T ; U^{*}\right), 1 / p+1 / q=1$, with $1<p<\infty$. As we have seen, this is an 'abstract trace theory' result, more amenable to show than (3.2a) in the case of mixed problems for partial differential equations, see [L-L-T.1, L-T.6] for second order hyperbolic equations and [L.3, L-T.7, L-T.8] for Euler-Bernoulli equations.

REMARK 3.2. As in step 3 of the proof of the Theorem in $\S 1$, assumption $\left(\mathrm{H}_{2}\right)$ implies that the operator

$$
\left\{\begin{array}{l}
(L u)(t)=\int_{0}^{t} e^{A(t-\tau)} B u(\tau) d \tau \\
\text { is continuous } L^{p}(0, T ; U) \rightarrow C([0, T] ; X) ;
\end{array}\right.
$$

i.e. there exists a constant $k_{T}>0$ such that

$$
\max _{0 \leq t \leq T}\|(L u)(t)\|=\max _{0 \leq t \leq T}\left\|\int_{0}^{t} e^{A(t-\tau)} B u(\tau) d \tau\right\| \leq k_{T}\left\{\int_{0}^{T}\|u(t)\|^{p} d t\right\}^{1 / p}
$$


a fact which we shall use below. We have also seen in step 1 of the proof of the Theorem in $\S 1$ that, conversely, the property that $L$ is continuous $L^{p}(0, T ; U) \rightarrow$ $L^{p}(0, T ; X)$ implies condition (3.2a) when $A$ is a group generator. Note also that for $t<T$ we have by a change of variable: $(L u)(t)=(L \mu)(T)$, where $\mu(\tau)=u(t-T+\tau)$ for $T-t<\tau<T$ and $\mu(\tau) \equiv 0$ for $0<\tau<T-t$.

THEOREM. Under the above assumptions $\left(\mathrm{H}_{1}\right)$ and $\left(\mathrm{H}_{2}\right)$, the operator $L$ defined by (3.3a) is continuous $L^{p}(0, \infty ; U) \rightarrow L^{p}(0, \infty ; X), 1<p<\infty$. The case $p=1$ is also included under assumption (3.3b).

ProOF, STEP 1. We let $f(t)=\|(L u)(t)\|^{p}$ and obtain

$$
\int_{0}^{\infty}\|(L u)(t)\|^{p} d t=\int_{0}^{\infty} f(t) d t=\sum_{n=0}^{\infty} \int_{n T}^{(n+1) T} f(t) d t=\sum_{n=0}^{\infty} \int_{0}^{T} f(n T+t) d t .
$$

By splitting the interval $[0, n T+t]$ in $[0, T],[T, 2 T]$, etc. and a change of variable, we compute for $n=1,2, \ldots$

$$
\begin{aligned}
f(n T+t)= & \left\|\int_{0}^{n T+t} e^{A(n T+t-\tau)} B u(\tau) d \tau\right\|^{p} \\
= & \| \sum_{j=1}^{n} e^{A((n-j) T+t)} \int_{0}^{T} e^{A(T-\tau)} B u((j-1) T+\tau) d \tau \\
& +\int_{0}^{t} e^{A(t-\tau)} B u(n T+\tau) d \tau \|^{p}
\end{aligned}
$$

(using (3.1) and (3.3b))

$$
\begin{aligned}
& \leq k_{T}^{p}\left\{e^{-\omega t} \sum_{j=1}^{n} e^{-\omega(n-j) T}\left(\int_{0}^{T}\|u((j-1) T+\tau)\|^{p} d \tau\right)^{1 / p}\right. \\
& \left.\quad+\left(\int_{0}^{T}\|u(n T+\tau)\|^{p} d \tau\right)^{1 / p}\right\}^{p} \\
& \leq k_{T}^{p} c_{p}\left\{e^{-\omega p t}\left(\sum_{j=1}^{n} e^{-\omega(n-j) T}\|u\|_{L^{p}((j-1) T, j T ; U)}\right)^{p}+\|u\|_{L^{p}(n T,(n+1) T ; U)}^{p}\right\}
\end{aligned}
$$

where in the last step we have used $(x+y)^{p} \leq c_{p}\left(x^{p}+y^{p}\right)$ for $0 \leq x, y<\infty$ where $c_{p}$ is a constant depending on $p$, but not on $x, y[\mathrm{D}-\mathrm{S}, \mathrm{p} .120]$. We now set

$$
v_{j}=\left\{\int_{(j-1) T}^{j T}\|u(\tau)\|^{p} d \tau\right\}^{1 / p}, \quad \text { so that } \sum_{j=1}^{\infty} v_{j}^{p}=\|u\|_{L^{p}(0, \infty ; U)}^{p}
$$


By Hölder inequality with $1 / p+1 / q=1$

$$
\begin{aligned}
\sum_{j=1}^{n} e^{-\omega(n-j) T} v_{j} & =\sum_{j=1}^{n} e^{-\omega(n-j) T / q} e^{-\omega(n-j) T / p} v_{j} \\
& \leq\left\{\sum_{j=1}^{n} e^{-\omega(n-j) T}\right\}^{1 / q}\left\{\sum_{j=1}^{n} e^{-\omega(n-j) T} v_{j}^{p}\right\}^{1 / p} \\
& \leq\left(\frac{1}{1-e^{-\omega T}}\right)^{1 / q}\left\{\sum_{j=1}^{n} e^{-\omega(n-j) T} v_{j}^{p}\right\}^{1 / p}
\end{aligned}
$$

Thus, by (3.6)-(3.8), we obtain for $n=1,2, \ldots$

$$
f(n T+t) \leq C_{p, \omega, T}\left\{e^{-\omega p t} \sum_{j=1}^{n} e^{-\omega(n-j) T} v_{j}^{p}+v_{n+1}^{p}\right\}
$$

where $C_{p, \omega, T}$ is a constant depending on $p, \omega, T, k_{T}$. Then (3.9) implies

$$
\sum_{n=1}^{\infty} \int_{0}^{T} f(n T+t) d t \leq \text { Const }_{p, \omega, T}\left\{\sum_{n=1}^{\infty} \sum_{j=1}^{n} e^{-\omega(n-j) T} v_{j}^{p}+\sum_{n=1}^{\infty} v_{n+1}^{p}\right\}
$$

But one readily sees that

$$
\sum_{n=1}^{\infty} \sum_{j=1}^{n} e^{-\omega(n-j) T} v_{j}^{p}=\left(\sum_{k=0}^{\infty} e^{-k \omega T}\right) \sum_{j=1}^{\infty} v_{j}^{p}=\frac{1}{1-e^{-\omega T}}\|u\|_{L^{p}(0, \infty ; U)}^{p}
$$

by rearranging the (positive) terms of the double sum which form an infinite lower triangular matrix, and summing up along the diagonals of it. Thus, (3.4), (3.10), (3.11), (3.7) and the term $n=0: \int_{0}^{T} f(t) d t$ which satisfies (3.2b), imply that

$$
\int_{0}^{\infty}\|(L u)(t)\|^{p} d t \leq \mathrm{const}\|u\|_{L^{p}(0, \infty ; U)}^{p}
$$

as desired, where const depends on $k_{T}, T, p, \omega$.

REMARK 3.3. Adaptation of the duality argument in steps 3-4 in the proof of the Theorem in $\S 1$ permits likewise to show that $L$ is also continuous $L^{p}(0, \infty ; U) \rightarrow$ $C_{b}([0, \infty] ; X)$, where $C_{b}$ is the space of $X$-valued continuous functions which are uniformly bounded on $[0, \infty]$.

Examples covered by the Theorem of the present section, with $p=2$ in fact, include: (i) the hyperbolic dynamics (1.0) in Example 1 with the addition of a term $-\gamma y_{t}, \gamma=$ const $>0$ of viscous damping and (ii) the Euler-Bernoulli mixed problems as in [L-T.7, L-T.8] also with the addition of a term of viscous damping. 


\section{REFERENCES}

[C.1] S. Chang, Ph. D. dissertation, Mathematics Department, Univ. of Florida, 1984.

[C-L.1] S. Chang and I. Lasiecka, Riccati equations for nonsymmetric and nondissipative hyperbolic systems, J. Math. Anal. Appl. 115 (1986), 378-414.

[DaP-L-T.1] G. Da Prato, I. Lasiecka and R. Triggiani, A direct study of the Riccati equation arising in hyperbolic boundary control problems, J. Differential Equations 64 (1986), 26-47.

[D-S.1] N. Dunford and J. T. Schwartz, Linear operators. Part I, Interscience, New York, 1958.

[F-L-T.1] F. Flandoli, I. Lasiecka and R. Triggiani, Algebraic Riccati equations with non-smoothing observation arising in hyperbolic and Euler-Bernoulli equations, Ann. Mat. Pura Appl. (to appear).

[H.1] F. L. Ho, Observabilité frontiere de l'equation des ondes, C. R. Acad. Sci. Paris 302 (1986).

[H-P.1] E. Hille and R. Phillips, Functional analysis and semigroups, Amer. Math. Soc. Colloq. Publ., vol. 31, Amer. Math. Soc., Providence, R.I., 1957.

[L.1] J. L. Lions, Controle des systemes distribues singuliers, Gauthier-Villars, 1983.

[L.2] — Controlabilité exacte des systemes distribues, C. R. Acad. Sci. Paris 302 (1986), 471-475.

[L.3] _ Optimal control of systems governed by partial differential equations, Springer-Verlag, Berlin and New York, 1971.

[L.4] J.-L. Lions, Un resultat de regularité (paper dedicated to S. Mizohata), Current Topics in Partial Differential Equations, Y. Ohya et al., Eds., Kinokuniya, Tokyo, 1986.

[L-L-T.1] I. Lasiecka, J. L. Lions and R. Triggiani, Non homogeneous boundary value problems for second order hyperbolic operators, J. Math. Pures Appl. 69 (1986), 149-192.

[L-M.1] J. L. Lions and E. Magenes, Nonhomogeneous boundary value problems and applications, vols. I, II, Springer-Verlag, Berlin and New York, 1972.

[L-T.1] I. Lasiecka and R. Triggiani, A cosine operator approach to modeling $L^{2}\left(0, T ; L^{2}(\Gamma)\right)-$ boundary input hyperbolic equations, Appl. Math. Optim. 7 (1981), 35-93.

[L-T.2] _ Regularity of hyperbolic equations under $L^{2}\left(0, T ; L^{2}(\Gamma)\right)$-boundary terms, Appl. Math. Optim. 10 (1983), 275-286.

[L-T.3] — Riccati equations for hyperbolic partial differential equations with $L^{2}\left(0, T ; L^{2}(\Gamma)\right)$ Dirichlet boundary terms, SIAM J. Control Optim. 24 (1986), 884-926.

[L-T.4] _ Uniform exponential energy decay of wave equation in a bounded region with $L^{2}(0, \infty$; $\left.L^{2}(\Gamma)\right)$-feedback control in the Dirichlet boundary conditions, J. Differential Equations 65 (1986), 340-390.

[L-T.5] __, Hyperbolic equations with nonhomogeneous Neumann boundary terms. Part I: Regularity, preprint 1983.

[L-T.6] _ Sharp regularity results for hyperbolic equations of Neumann type, 1987.

[L-T.7] _ Regularity theory for a class of Euler Bernoulli equations: a cosine operator approach, Boll. Un. Mat. Ital. (to appear).

[L-T.8] _ Exact controllability of the Euler-Bernoulli equation with controls in the Dirichlet and Neumann boundary conditions: a non-conservative case, SIAM J. Control \& Optim. (to appear).

[L-T.9] __ Infinite horizon quadratic cost problems for boundary control problems, Proc. 20th CDC Conference, pp. 1005-1010, Los Angeles, December 1987.

[R.1] J. Rauch, $L^{2}$ is a continuable initial condition for Kreiss' mixed problems, Comm. Pure Appl. Math. 25 (1972), 265-285.

[R.2] D. L. Russell, Controllability and stabilizability theory for linear partial differential equations: recent progress and open questions, SIAM Rev. 20 (1978), 639-740.

[K.1] H. O. Kreiss, Initial boundary value problem for hyperbolic systems, Comm. Pure Appl. Math. 13 (1970), 277-298.

[T.1] R. Triggiani, A cosine operator approach to modeling boundary inputs problems for hyperbolic systems, Lecture Notes in Math., vol. 6, Springer-Verlag, Berlin and New York, 1978, pp. 380-390.

[T.2] _ Exact boundary controllability on $L^{2}(\Omega) \times H^{-1}(\Omega)$ for the wave equation with Dirichlet control acting on a portion of the boundary, and related problems, Appl. Math. Optim. (to appear), Springer-Verlag Lecture Notes in Control Sciences, vol. 102, pp. 292-332, Proceedings of 3rd International Conference, Vorau, Austria, July 6-12, 1986 . 
[T.3] _ Wave equation on a bounded domain with boundary dissipation: an operator approach, $\mathbf{J}$. Math. Anal. Appl. (to appear); also, Lecture Notes in Pure and Applied Mathematics, vol. 108, pp. 283-310; in Operator Methods for Optimal Control Problems (Sung J. Lee, Ed.), Marcel Dekker (1987); also in Recent Advances in Communication and Control Theory, honoring the sixtieth anniversary of A. V. Balakrishnan (R. E. Kalman and G. I. Marchuk, Eds.), Optimization Software (New York, 1987), pp. 262-286.

[W.1] G. Weiss, Admissibility of unbounded control operators, preprint.

Department of Applied Mathematics, Thornton Hall, University of Virginia, Charlottesville, Virginia 22903 